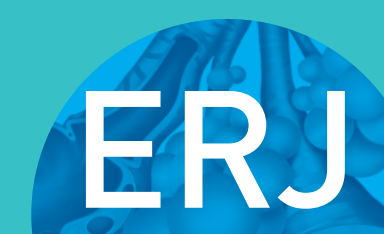

open research

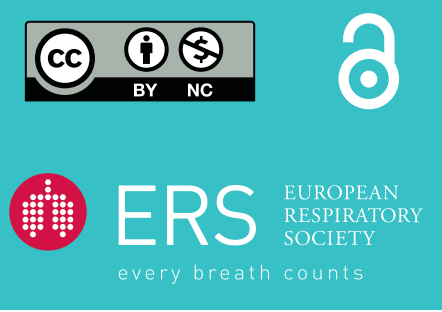

\section{Core outcome set for the management of acute exacerbations of chronic obstructive pulmonary disease: the COS- AECOPD ERS Task Force study protocol}

Alexander G. Mathioudakis (1) 1,2,37, Fekri Abroug ${ }^{3}$, Alvar Agusti $^{4,5}$, Per Bakke 6 , Konstantinos Bartziokas ${ }^{7}$, Bianca Beghe ${ }^{8}$, Andras Bikov ${ }^{1,2}$, Thomas Bradbury ${ }^{9}$, Guy Brusselle (10 ${ }^{10,11}$, Cordula Cadus ${ }^{12,13}$, Courtney Coleman ${ }^{14}$, Marco Contoli ${ }^{15}$, Alexandru Corlateanu (1016, Olga Corlateanu ${ }^{17}$, Gerard Criner ${ }^{18}$, Balazs Csoma ${ }^{19}$, Alexander Emelyanov ${ }^{20}$, Rosa Faner ${ }^{4}$, Gustavo Fernandez Romero ${ }^{18}$, Zeineb Hammouda ${ }^{3}$, Peter Horváth ${ }^{19}$, Arturo Garcia Huerta ${ }^{4,21}$, Michael Jacobs ${ }^{18}$, Christine Jenkins ${ }^{9}$, Guy Joos (11) ${ }^{11}$, Olga Kharevich ${ }^{22}$, Konstantinos Kostikas ${ }^{7}$, Elena Lapteva ${ }^{22}$, Zsofia Lazar ${ }^{19}$, Joerg D. Leuppi ${ }^{12,13}$, Carol Liddle ${ }^{14}$, Alejandra López-Giraldo ${ }^{4,5}$, Vanessa M. McDonald ${ }^{23,24,25}$, Rune Nielsen ${ }^{6,26}$, Alberto Papi $\mathbb{1}^{15}$, Isabel Saraiva ${ }^{14}$, Galina Sergeeva $^{20}$, Agni Sioutkou ${ }^{7}$, Pradeesh Sivapalan ${ }^{27,28}$, Elizabeth Stovold ${ }^{29}$, Hao Wang ${ }^{30}$, Fuqiang Wen ${ }^{30}$, Janelle Yorke 31,32 , Paula R. Williamson ${ }^{33,34}$, Jørgen Vestbo (1) 1,2 and Jens-Ulrik Jensen $27,35,36,37$

ABSTRACT Randomised controlled trials (RCTs) on the management of COPD exacerbations evaluate heterogeneous outcomes, often omitting those that are clinically important and patient relevant. This limits their usability and comparability. A core outcome set (COS) is a consensus-based minimum set of clinically important outcomes that should be evaluated in all RCTs in specific areas of health care. We present the study protocol of the COS-AECOPD ERS Task Force, aiming to develop a COS for COPD exacerbation management, that could remedy these limitations.

For the development of this COS we follow standard methodology recommended by the COMET initiative. A comprehensive list of outcomes is assembled through a methodological systematic review of the outcomes reported in relevant RCTs. Qualitative research with patients with COPD will also be conducted, aiming to identify additional outcomes that may be important to patients, but are not currently addressed in clinical research studies. Prioritisation of the core outcomes will be facilitated through an extensive, multi-stakeholder Delphi survey with a global reach. Selection will be finalised in an international, multi-stakeholder meeting. For every core outcome, we will recommend a specific measurement instrument and standardised time points for evaluation. Selection of instruments will be based on evidence-informed consensus.

Our work will improve the quality, usability and comparability of future RCTs on the management of COPD exacerbations and, ultimately, the care of patients with COPD. Multi-stakeholder engagement and societal support by the European Respiratory Society will raise awareness and promote implementation of the COS.

$@$ ERSpublications

The COS-AECOPD @EuroRespSoc Task Force is developing a core outcome set (COS) for \#COPD exacerbation management. COS is an agreed minimum set of clinically important outcomes to be evaluated in all RCTs and can improve their usability and comparability. https://bit.ly/3ftAXk6

Cite this article as: Mathioudakis AG, Abroug F, Agusti A, et al. Core outcome set for the management of acute exacerbations of chronic obstructive pulmonary disease: the COS-AECOPD ERS Task Force study protocol. ERJ Open Res 2020; 6: 00193-2020 [https://doi.org/10.1183/23120541.00193-2020].

Protocol Registration: COMET database ID: 1325

Received: 17 April 2020 | Accepted after revision: 15 June 2020

Copyright $\odot$ ERS 2020. This article is open access and distributed under the terms of the Creative Commons Attribution Non-Commercial Licence 4.0. 


\section{Introduction}

COPD, the third leading cause of death globally, has a growing prevalence and currently affects over 174 million people [1-3]. Acute exacerbations punctuate the natural history of COPD, determining disease morbidity, mortality and progression [3-5]. Every year, up to $40 \%$ of patients diagnosed with COPD have at least one moderate or severe exacerbation, while $9-16 \%$ experience more [6, 7]. As a result, exacerbations are responsible for a significant proportion of all hospital admissions (one in eight in the United Kingdom), while the 90-day mortality rate of an admission for an exacerbation exceeds $15 \%$ [8-10]. Exacerbations are also associated with a substantial socioeconomic burden $[3,10]$.

While our understanding of the pathogenesis and underlying mechanisms of exacerbations is growing rapidly $[5,11,12]$, their management remains only partly effective and almost unchanged for decades $[3,8,10]$. Standard treatment still consists of three main components; bronchodilators, antibiotics and corticosteroids. Therefore, novel treatments are to be expected, and an increasing number of clinical research studies will be conducted in the coming years. These will include randomised controlled trials (RCTs) that can provide conclusive evidence of the safety and effectiveness of an intervention, by minimising potential biases [13, 14]. Clinical trials seek to evaluate the safety, efficacy and/or clinical effectiveness of interventions by comparing their effects on outcomes. Only a limited number of outcomes can be evaluated in each trial. Researchers often select outcomes that are easier to measure, require fewer resources, are more likely to favour one intervention over the other(s), or address specific hypotheses, which may be of limited importance to patients, clinicians or the regulatory authorities [15]. Consequently, crucial data on potential beneficial or harmful effects of interventions are often missed. This hampers the interpretability and potential value of RCTs, whose main aim is to inform clinical guidelines and practice. Moreover, the use of different instruments to evaluate the same outcome is likely to limit comparability.

Particularly in trials evaluating the management of COPD exacerbations, the definition of outcomes is still vague and heterogeneous, while consistent use of relevant, comparable, patient important outcomes is lacking. In a recent methodological systematic review [16, 17], we found significant heterogeneity in the

Affiliations: ${ }^{1}$ Division of Infection, Immunity and Respiratory Medicine, School of Biological Sciences, The University of Manchester, Manchester Academic Health Science Centre, Manchester, UK. ${ }^{2}$ The North West Lung Centre, Wythenshawe Hospital, Manchester University NHS Foundation Trust, Manchester, UK. ${ }^{3} \mathrm{Hospital}$ Fattouma Bourguiba, Monastir, Tunisia. ${ }^{4}$ Institut d'Investigacions Biomèdiques August Pi i Sunyer (IDIBAPS), National Spanish Network for Respiratory Research (CIBERES), Barcelona, Spain. ${ }^{5}$ Respiratory Institute, Hospital Clinic, University of Barcelona, Barcelona, Spain. ${ }^{6}$ Department of Clinical Science, University of Bergen, Bergen, Norway. ${ }^{7}$ Respiratory Medicine Dept, University of loannina School of Medicine, loannina, Greece. ${ }^{8}$ Section of Respiratory Diseases, Dept of Medicine, University of Modena and Reggio Emilia, Modena, Italy. ${ }^{9}$ The George Institute for Global Health, University of New South Wales, Sydney, Australia. ${ }^{10}$ Depts of Epidemiology and Respiratory Medicine, Erasmus Medical Center, Rotterdam, The Netherlands. ${ }^{11}$ Dept of Respiratory Medicine, Ghent University Hospital, Ghent, Belgium. ${ }^{12}$ University Department of Medicine, Cantonal Hospital Basell and Liestal, Basell, Switzerland. ${ }^{13}$ Faculty of Medicine, University of Basel, Basel, Switzerland. ${ }^{14}$ European Lung Foundation, Sheffield, UK. ${ }^{15}$ Research Center on Asthma and COPD, Dept of Medical Sciences, University of Ferrara, Ferrara, Italy. ${ }^{16}$ Dept of Respiratory Medicine, State University of Medicine and Pharmacy "Nicolae Testemitanu”, Chisinau, Moldova. ${ }^{17}$ Department of Internal Medicine, State University of Medicine and Pharmacy "Nicolae Testemitanu", Chisinau, Moldova. ${ }^{18}$ Dept of Thoracic Medicine and Surgery, Lewis Katz School of Medicine at Temple University, Philadelphia, PA, USA. ${ }^{19}$ Dept of Pulmonology, Semmelweis University, Budapest, Hungary. ${ }^{20}$ Dept of Respiratory Medicine and Allergy, NorthWestern Medical University, St Petersburg, Russian Federation. ${ }^{21}$ Respiratory Intensive Care Division, Clinica Sagrada Familia, Barcelona, Spain. ${ }^{22}$ Dept of Pulmonology and Tuberculosis, Belarusian Medical Academy of Postgraduate Education, Minsk, Belarus. ${ }^{23}$ Dept of Respiratory and Sleep Medicine, Medical and Interventional Services, John Hunter Hospital, Hunter New England Local Health District, New Lambton Heights, Australia. ${ }^{24}$ Priority Research Centre for Healthy Lungs, University of Newcastle, Callaghan, Australia. ${ }^{25}$ School of Nursing and Midwifery, University of Newcastle, Callaghan, Australia. ${ }^{26}$ Dept of Thoracic Medicine, Haukeland University Hospital, Bergen, Norway. ${ }^{27}$ Section of Respiratory Medicine, Dept of Internal Medicine, HerlevGentofte Hospital, Hellerup, Denmark. ${ }^{28}$ Dept of Internal Medicine, Zealand University Hospital, Roskilde, Denmark. ${ }^{29}$ Cochrane Airways Group, Population Health Research Institute, St George's University of London, London, UK. ${ }^{30}$ Dept of Respiratory and Critical Care Medicine, West China Hospital/West China School of Medicine, Sichuan University, Chengdu, China. ${ }^{31}$ School of Health Sciences, Faculty of Biology, Medicine and Health, University of Manchester, Manchester, UK. ${ }^{32}$ Christie Patient Centred Research, The Christie NHS Foundation Trust, Manchester, UK. ${ }^{33}$ Liverpool Clinical Trials Centre, Dept of Biostatistics, University of Liverpool, Liverpool, UK. ${ }^{34} \mathrm{MRC}$ North West Hub for Trials Methodology Research, Institute of Translational Medicine, University of Liverpool, Liverpool, UK. ${ }^{35} \mathrm{CHIP}$ and PERSIMUNE, Dept of Infectious Diseases, Rigshospitalet, Copenhagen, Denmark. ${ }^{3}$ Institute of Clinical Medicine, Faculty of Health Sciences, University of Copenhagen, Copenhagen, Denmark. ${ }^{37} \mathrm{Co}$-chairs of the COS-AECOPD ERS Task Force.

Correspondence: Jørgen Vestbo, Division of Infection, Immunity and Respiratory Medicine, School of Biological Sciences, The University of Manchester, Southmoor Road, Manchester, M23 9LT, UK. E-mail: jorgen.vestbolamanchester.ac.uk 


\begin{tabular}{|c|c|c|}
\hline \multirow[t]{2}{*}{ Outcomes } & \multicolumn{2}{|c|}{ Frequency of reporting } \\
\hline & RCTs & SRs \\
\hline \multicolumn{3}{|l|}{ Patient important outcomes } \\
\hline Mortality & $101(82 \%)$ & $29(76 \%)$ \\
\hline Treatment success or failure & $77(63 \%)$ & $29(76 \%)$ \\
\hline Adverse effects & 73 (59\%) & $26(68 \%)$ \\
\hline Health status, symptoms \& quality of life & $73(59 \%)$ & $17(45 \%)$ \\
\hline Duration of exacerbations & $42(34 \%)$ & $20(53 \%)$ \\
\hline Re-exacerbation, re-hospitalisation & $33(27 \%)$ & $16(42 \%)$ \\
\hline Exercise capacity & $14(11 \%)$ & $1(3 \%)$ \\
\hline Anxiety and depression & $6(5 \%)$ & $1(3 \%)$ \\
\hline \multicolumn{3}{|c|}{ Surrogate, physiological and laboratory outcomes } \\
\hline Lung function & $58(47 \%)$ & $18(47 \%)$ \\
\hline Arterial blood gases and oxygen saturation & $40(33 \%)$ & $5(13 \%)$ \\
\hline Microbiological response & $16(13 \%)$ & $7(18 \%)$ \\
\hline Biomarkers & $32(26 \%)$ & $2(5 \%)$ \\
\hline Medication use & $18(15 \%)$ & $3(8 \%)$ \\
\hline
\end{tabular}

outcomes assessed and reported by trials on the management of COPD exacerbations (table 1). Only 63\% of all RCTs conducted during the last decade assessed the proportion of patients whose exacerbations were successfully treated or experience treatment failure, while less than $35 \%$ evaluated duration of the exacerbation as an outcome (either the duration of a hospital admission or symptoms). Finally, there was significant heterogeneity in the definition of outcomes and in the instruments used to evaluate them. This lack of standardisation complicates interpreting, comparing, contrasting and synthesising the results of RCTs. Thus, several recent meta-analyses on the management of COPD exacerbations have reported limited certainty in the available evidence [18-20].

We report the study protocol of the COS-AECOPD study, which will be conducted to address these limitations. We aim to develop a core outcome set (COS) to be used for RCTs evaluating the management of COPD exacerbations. The aim of a COS is to develop global, multi-stakeholder consensus on a minimum number of outcomes that future, relevant RCTs should measure and report on. It has been demonstrated that when COS are implemented, they homogenise the design of RCTs, increase their usability and comparability [21,22]. Additionally, a COS for COPD exacerbations will improve the possibilities for meaningful and statistically sound meta-analyses, helping to inform future clinical practice guidelines.

This project is supported by the European Respiratory Society (ERS Task Force 2019-12) and the European Lung Foundation (ELF). It is also supported by the DECODE-NET (DisEntangling Chronic Obstructive pulmonary Disease Exacerbations clinical trials NETwork), an emerging clinical trials network involving over 30 centres in Africa, Asia, Australia, Europe and America [23]. The DECODE-NET intends to use the resulting COS in planned and future trials.

\section{Methods}

The Core Outcome Measures in Effectiveness Trials (COMET), an initiative aiming to bring together people interested in the development and application of COS, has developed explicit methodology for the development of COS. For the development and reporting of this COS, we follow explicit methodology suggested by the COMET initiative (the COMET handbook), Core Outcome Set, STAndards for Development (COS-STAD), Core Outcome Set, STAndards for Reporting (COS-STAR) and Core Outcome Set, STAndardised Protocol Items (COS-STAP) documents (table 2) [24-27], which has already been implemented successfully in several high-quality COS projects [28-30]. COMET recommends the development of a comprehensive list of relevant outcomes followed by a prioritisation process, aimed to select the most important (core) outcomes. The long list of outcomes is formed by a methodological systematic review evaluating outcomes measured and reported in relevant RCTs. We have recently completed the systematic review [17]; results are summarised in table 1. It is recommended that the list of outcomes should also be informed by qualitative research aiming to elicit factors that are considered 
TABLE 2 Compliance of the COS-AECOPD with the COS-STAD standards for COS development

\begin{tabular}{|c|c|c|}
\hline Domain & Standard & COS-AECOPD \\
\hline \multirow[t]{4}{*}{ Scope } & Setting & Clinical research, focusing on RCTs \\
\hline & Condition & Treatment of acute exacerbations of COPD \\
\hline & Population & Adults with COPD exacerbations of any severity \\
\hline & Interventions & Any intervention \\
\hline \multirow[t]{3}{*}{$\begin{array}{l}\text { Stakeholder } \\
\text { involvement }\end{array}$} & Users & $\begin{array}{l}\text { Clinical researchers, trialists, guideline } \\
\text { developers, policy makers, regulators, } \\
\text { research funders, and the pharmaceutical } \\
\text { industry (with global representation) }\end{array}$ \\
\hline & Healthcare professionals & $\begin{array}{l}\text { Doctors, nurses, physiotherapists with } \\
\text { expertise in COPD (global representation) }\end{array}$ \\
\hline & Patients & $\begin{array}{l}\text { Patients with COPD and their carers (with } \\
\text { global representation) }\end{array}$ \\
\hline \multirow[t]{4}{*}{$\begin{array}{l}\text { Consensus } \\
\text { process }\end{array}$} & $\begin{array}{l}\text { Initial list of outcomes to be } \\
\text { informed by patients' and health } \\
\text { professionals' views }\end{array}$ & $\begin{array}{l}\text { Patients' and healthcare professionals' views } \\
\text { will be identified through methodological } \\
\text { systematic reviews and an extensive Delphi } \\
\text { study. Patients' views will also be captured } \\
\text { through qualitative studies }\end{array}$ \\
\hline & $\begin{array}{l}\text { A priori scoring process and } \\
\text { consensus definition }\end{array}$ & See Methods: Delphi survey \\
\hline & $\begin{array}{l}\text { A priori criteria for including, } \\
\text { dropping or adding outcomes. }\end{array}$ & See Methods: Delphi survey \\
\hline & $\begin{array}{l}\text { Avoid ambiguity of language used in } \\
\text { the list of outcomes }\end{array}$ & $\begin{array}{l}\text { The phrasing of the outcomes will be reviewed } \\
\text { by the COS panel and the patients } \\
\text { participating in the COS focus groups }\end{array}$ \\
\hline
\end{tabular}

relevant and important to patients and their caregivers, that may not be evaluated in clinical research studies. Prioritisation of the core outcomes is facilitated by a Delphi survey involving multiple stakeholders, with a global reach and a consensus meeting with similar characteristics.

\section{Study oversight}

This study will be conducted by the COS-AECOPD ERS Task Force. A steering committee was formed consisting of Alexander G. Mathioudakis, Jens-Ulrik Jensen, Jørgen Vestbo (clinical researchers with expertise in trials focusing on COPD exacerbations), Carol Liddle (patient representative) and Paula Williamson (COMET chair). The steering committee is responsible for the management and coordination of the study. The steering committee will meet every 3 months (face to face or via teleconference), to review the study progress, ensure the study complies with good clinical practice principles, relevant regulations and adheres to the study protocol.

\section{Qualitative research}

We will conduct a focus group discussion, followed by semi-structured interviews, aiming to identify outcomes that patients with COPD consider important. We will involve geographically spread participants with different disease severity, age, cultural and socioeconomic backgrounds. More specifically, participants will include male and female adults suffering from COPD, who have a history of a hospitalised exacerbations or frequent moderate exacerbations (treated in the community), or exacerbations with concomitant hypercapnic respiratory failure requiring noninvasive ventilation, during the preceding year. Some participants will be approached while recovering from an exacerbation, and others during stable disease state. Members of our team will recruit and interview patients in several countries with different socioeconomic characteristics, across the globe (we plan to recruit patients from Africa, Americas, Asia, Australia and Europe). Each participant will only be able to attend either one interview or the focus group meeting.

A focus group and semi-structured interviews using open-ended questions will be used to elicit participants' expectations and concerns regarding COPD exacerbations and their views on the outcomes of exacerbations. These questions were developed by academic and lay members (patients diagnosed with COPD) of our research team, with input from a qualitative researcher (JY), the COMET initiative chair (PW) and the COMET qualitative research team. They are summarised in table 3. Some of the participants will also 
TABLE 3 Pre-specified questions that will be used to facilitate the qualitative research

(semi-structured interviews) and the focus group

Pre-selected open-ended questions

Can you tell us about your experience of having an exacerbation (flare-up) of your COPD?

When you have a flare-up of your COPD, how does this impact your life? ${ }^{\#}$

What treatments were you offered for your recent exacerbations? How do medicines for your flare-ups make you feel? (Ask specifically for NIV) ${ }^{\#}$

To what extent the effects of treatments you had for exacerbations matched your expectations?

What specifically have you hoped from the treatments for your COPD exacerbations? ${ }^{\#}$

What concerns do you have about your future exacerbations (flare-ups)?

What are the most concerning effects of exacerbations in your life?

If a new treatment became available for COPD flare-ups, what specific effects would you like it to have on you? \# $^{\circ}$

Provide plain language description of the outcomes. Make sure the participants understand what an outcome is: Which outcomes do you think are important to be evaluated?

Were there any outcomes that you considered important previously that were not mentioned during this interview?

NIV: noninvasive ventilation. ${ }^{\#}$ : Ask specifically about physical/mental/social wellbeing.

contribute to the development of plain language descriptions of the identified outcomes. Think-aloud techniques will be used to evaluate how they interpret the outcomes [31]. Qualitative studies will be conducted in the language spoken in each participating country. All investigators who will contribute to the interviews will receive relevant material and/or a short introductory training presentation, to strengthen relevant skills and ensure consistency across the different study sites.

The focus group and all interviews will be audio recorded and anonymised. All outcomes described directly or indirectly by participants will be extracted verbatim, grouped and translated in the English language. The frequency that every outcome is volunteered by participants will be presented as a relative measure of importance of the outcome. In addition, we will undertake thematic analysis with a framework approach to data organisation [32], aiming to identify participants' hopes and concerns regarding COPD exacerbations and their treatment. We will explore differences in the responses of participants from different geographic and socioeconomic backgrounds.

\section{Delphi survey}

A Delphi survey is a widely used method to develop consensus. Participants are asked to complete several rounds of a questionnaire. In each round, participants are asked to review and consider adjusting their responses, considering the aggregated responses of all participants during the preceding round [33]. An international, multi-stakeholder Delphi survey will be conducted, to facilitate prioritisation of the core outcomes, among those identified through the methodological systematic reviews and qualitative research. We will invite representatives of four stakeholder groups to participate in the survey:

a) Patients diagnosed with COPD, who have experienced exacerbations, caregivers of such patients, or representatives of such patients (e.g. patient organisations)

b) Health professionals caring for patients (e.g. doctors, nurses, physiotherapists)

c) Clinician researchers (health professionals who care for patients but are also involved in designing research studies)

d) Other stakeholders, including regulators, policy makers, funders, guideline developers or those working in health technology assessment organisations.

Participants will be informed about the rationale and importance of COS development and that they will be required to respond to two Delphi rounds. We will use DelphiManager, a secure software developed by the COMET initiative. The survey will be available in English and at least three other languages, which will be selected with the aim of maximising the reach of the survey and based on the available resources. Two-way translations will be prepared by members of our research team, in collaboration with the ELF, and input from lay members of their COPD Patient Advisory Group.

We will use a modified Delphi approach proposed by the COMET initiative, involving two Delphi rounds [34]. In the first Delphi round, all unique outcomes identified through systematic reviews and qualitative research will be listed in scientific and plain language, and we will ask participants to rate the importance of every outcome. We will also encourage participants to suggest additional outcomes they 
consider relevant. Any genuinely new outcomes (not overlapping with previously identified outcomes) suggested by the respondents will be included in the subsequent Delphi round. In the next round, graphical displays of the scores submitted from each stakeholder group during the first round will be presented to the participants, who will be asked to rate the importance of the outcomes for a second time (figure 1). While the aim of this survey will be to reach consensus, participants will not be under any pressure to change their ratings if they do not want to.

In both rounds, participants will be asked to rate the importance of each outcome for clinical decision making on a scale from 1 to 9, following guidance by GRADE ( Grading of Recommendations, Assessment, Development and Evaluation). Scores between 1-3 signify an outcome of limited importance, 4-6 signify an important but not critical outcome and 7-9 signify a critical outcome $[35,36]$. Answers will be sought by all stakeholder groups and responses by each group will be analysed separately, in each Delphi round. Consensus will be defined after the second Delphi round, using the following methodology: outcomes rated between $7-9$ (critical) by $\geqslant 70 \%$ and between $1-3$ (of limited importance) by $\leqslant 15 \%$ of all participants in each stakeholder group, will be selected for inclusion in the COS. In parallel, outcomes that are rated between $7-9$ (critical) by $\leqslant 50 \%$ of all participants in each stakeholder group will be excluded from the COS.

We have conducted extensive preparatory work to achieve a global reach of the survey. Invitations will be sent to:

a) Members of the ERS with a documented interest in airway diseases.

b) Patients with COPD and their caregivers through the ELF's network of local and national patient organisations representing patients across the world. Also, patients diagnosed with COPD who have subscribed to the UK's National Institute for Health Research (NIHR) Research for the Future Initiative and other similar organisations facilitating patients' recruitment.

c) Clinical researchers, policy makers, guideline developers, regulators, research funders and industry representatives from all continents who have published on COPD exacerbations during the last decade and their emails were identified through extensive literature searches ( $>5000$ unique emails).

For all these stakeholders, we have developed invitations that are compliant with the General Data Protection Regulation and e-Privacy regulations.

\section{Outcome measure instruments and timing}

Outcome measure instruments refer to the specific methodology used to evaluate the impact of an intervention on an outcome. More specifically, while outcomes answer the question "What to measure?", instruments refer to the question "How to measure it?". For example, St George's Respiratory Questionnaire or the Clinical COPD Questionnaire are both instruments that assess the outcome health status in COPD [17]. Often, different instruments are used for evaluating the same outcome, limiting the
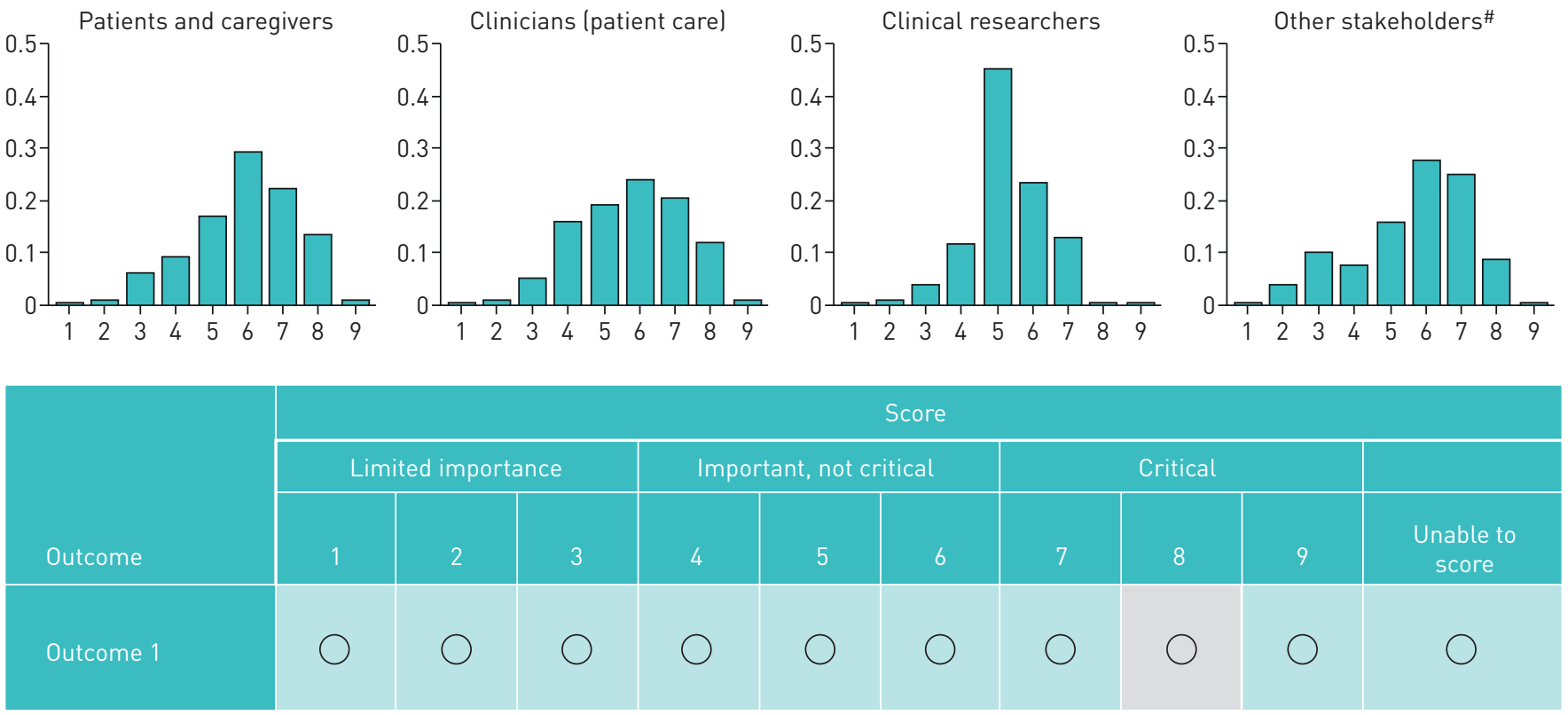

FIGURE 1 Delphi round 2. Respondents will be provided with the score distributions from the first Delphi round, stratified by stakeholder category. Their previous scoring will also be highlighted. (In this example, the respondent's score for this outcome was 8.) Respondents will be asked to reconsider their scoring, based on the available data. They will be under no pressure to change their scores. 
comparability of the RCT results. For this reason, we will recommend a specific instrument for each of the selected core outcomes.

The COnsensus-based Standards for the selection of health status Measurement Instruments (COSMIN) recommends a thorough methodology for in-depth evaluation and selection of outcome instruments [37]. However, such extensive methodological studies are beyond the scope and resources of the COS-AECOPD study. For this reason, we will follow a more pragmatic approach. For every candidate core outcome, we will identify the instruments that were used in the studies included in our methodological systematic review and we will note how frequently each instrument was used [17]. For outcomes that are often evaluated by the same instrument (in $>40 \%$ of the trials), we will strongly consider recommending this instrument, unless important methodological issues are raised by any of the task force panel members. In any other case, we will undertake focused literature searches of Medline/PubMed and the COSMIN database, to identify studies evaluating the quality of every instrument. Our task force panel will review available evidence and will provisionally prioritise the use of a single instrument after considering: 1) the frequency each instrument has been used in RCTs; 2) the time and resources required to assess each instrument; and 3) available data on their measurement properties as described by COSMIN recommendations (validity, reliability, responsiveness and interpretability).

Timing of outcomes evaluation is also crucial as COPD exacerbations are acute, dynamic events. Minimum timepoints for follow-up evaluation will be provisionally selected based on consensus among the panel members and in consultation with ELF's COPD Patient Advisory Group. To reach an informed consensus, the task force members will review the timings of outcome evaluation in previous trials and the impact of timing on the results.

Selection of the instruments and follow-up timepoints will be presented during the consensus meeting, feedback will be invited by all participants and the recommendations will be finalised based on consensus within the consensus group

\section{Consensus meeting}

A consensus meeting will take place during the ERS International Congress. Members of all previously described stakeholder groups will be invited, including at least five patient representatives. Geographic and socioeconomic diversity will be considered in the selection of participants.

During this meeting, results of the Delphi survey will be presented objectively, starting from all outcomes that reached consensus for inclusion, or exclusion. Then, all outcomes will be discussed. All stakeholder groups will be given the opportunity to comment and vote on the selection of the final, core outcomes. Our aim will be to select a small number of the most important outcomes, to ensure practicality of use of this COS. Relevant arguments for or against the inclusion of an outcome and the vote counts will be noted.

Next, results of the outcome instruments' evaluation will be discussed. The group will finalise the selection of a single instrument for every outcome and will decide on the recommended follow-up time points. Reasoning for all decisions will be described narratively in the final study report.

\section{Sample size}

We did not conduct formal power calculations for this study, as there are no strict recommendations on the number of participants in a qualitative study or Delphi survey. In the first stage (qualitative research), we will interview at least 70 patients, and until we are confident that saturation has been achieved and potential socioeconomic and geographic differences have been captured.

For the Delphi survey, we have developed a thorough strategy for recruiting members of each stakeholder category and we aim to engage as many participants as possible, to develop global, multi-stakeholder consensus, while also raising awareness about the issue and our COS. We anticipate a study population at the range of hundreds.

\section{Protocol registration}

The study protocol of the COS-AECOPD ERS Task Force has been registered prospectively with the COMET database (COMET ID: 1325).

\section{Discussion}

Comparability (and occasionally interpretability) of RCTs on COPD exacerbation management is particularly problematic [17]. The COS-AECOPD ERS Task Force aims to remedy these limitations by developing a COS for the management of COPD exacerbations, that will promote standardisation of the outcomes and their measurement instruments reported in future RCTs. 
COMET suggests that a qualitative systematic synthesis could occasionally replace patient interviews. Our qualitative review (unpublished data), yielded data from one previous systematic review and three primary studies. Fatigue and psychological wellbeing emerged as outcomes of importance to patients that are rarely tested in RCTs and systematic reviews and were included in the longlist of outcomes. However, confidence on the findings, evaluated using CERQual methodology [38], was low, because of concerns regarding the adequacy and relevance of the available data. Adequate understanding of patients' needs and priorities is crucial for the development of high-quality COS. Characteristically, qualitative studies conducted by the OMERACT (Outcome Measures in Rheumatology) almost two decades ago identified fatigue as a crucial outcome of rheumatoid arthritis [39]. Fatigue, which was previously not evaluated in RCTs, is currently one of the most frequently reported and informative outcomes in that field. For these reasons, we decided to conduct additional, original qualitative research to inform the COS-AECOPD.

For outcomes prioritisation, we decided to use the modified Delphi approach proposed by COMET. Instead of asking the respondents to identify potential outcomes, we will feed the survey with outcomes identified through intensive methodological and qualitative research and this will ensure that the longlist of outcomes is more complete. Respondents will also be encouraged to suggest additional relevant outcomes. Moreover, we will limit the number of Delphi rounds to two, to limit attrition. While two rounds may not be adequate to reach consensus for many of the outcomes, the results will be fed to a multi-stakeholder consensus group meeting for finalisation. This approach has been successfully utilised in previous high-quality COS [28-30].

A potential limitation of this study is our pragmatic approach towards the selection of outcome instruments and the follow-up timing, which will not fully adhere to the COSMIN recommendations. This is very unlikely to affect the selection of instruments for simple, objective outcomes (such as mortality). It might affect the selection of measures of composite or patient reported outcomes. However, selection of instruments will be informed by: 1) focused literature searches of studies evaluating the measurement properties of the instruments; 2) current standard research practice, as our aim is to enhance homogeneity across different RCTs; and 3) the experience of several principal investigators and methodologists involved in COPD exacerbations trials, who are involved in this task force. Moreover, while researchers will strongly be encouraged to use the core outcomes in all future RCTs, they will also be encouraged to measure other outcomes, that may be relevant to their interventions, RCTs or interests but may also include methodological evaluation of alternative instruments.

High resource requirements for the evaluation of certain outcomes included in COS may limit their implementation, especially in pilot or early phase studies. However, the outcomes identified through our systematic reviews are simple and inexpensive to measure and can be captured after a relatively short follow-up period [17]. In addition, costs and resource requirements will be considered by the panel when selecting the outcome instruments. Finally, it would not be expected that early phase studies would include the COS necessarily, however it may be important that they include some of the core outcomes, to gather data that would help power the later-phase studies.

The outcomes suggested by different regulatory bodies may not be included in the COS-AECOPD. In this case, investigators would be advised to evaluate both the outcomes required by the regulatory authorities and the additional outcomes that will be proposed by this COS, which will be informed by a global, multi-stakeholder agreement. It is anticipated that regulatory authorities are likely to start endorsing high-quality COS in the near future. For example, the US Food and Drug Administration is currently developing three COS in a pilot project (for other diseases) [40].

A major strength of the COS-AECOPD study is the strong design, which is based on a thorough methodological systematic review and extensive qualitative research to develop a longlist of clinically relevant outcomes and an extensive Delphi survey, aiming to develop consensus. Moreover, the global reach and involvement of all relevant stakeholders, following an exhaustive strategy to recruit and engage them, will facilitate the development of international consensus, improve awareness of the methodological issues and enhance the COS implementation in future research studies. Societal support by the ERS will also promote awareness and implementation.

The need for high-quality research on COPD exacerbations is prioritised by the ERS. Apart from this Task Force, the Society is also supporting the CICERO (Collaboration In COPD ExaceRbatiOns) ERS Clinical Research Collaboration, aiming to set up a pan-European, prospective observational cohort study of patients hospitalised with COPD exacerbations, to evaluate their clinical and mechanistic characteristics [41]. As part of this project the CICERO team is developing relevant methodology, including a comprehensive data collection plan for such studies. The two projects are developed in a collaborative fashion, where pertinent interim data of each project will be shared and used to inform the following steps of each project. 
Overall, it is our strong belief that the development of a COS for the management of COPD exacerbations will improve the quality, comparability and usability of future RCTs and will consequently have a positive impact on the management of COPD exacerbations, clinical practice guidelines and the care of patients with COPD.

Acknowledgement: We are thankful to the ERS and the ELF for supporting this study; to the COPD Patient Advisory Group of the ELF for reviewing the plain language material of our study; and to the COMET qualitative team, for providing input in the development of the patient interview questions.

Author contribution: A.G. Mathioudakis, J. Vestbo and J-U. Jensen conceived this study. A.G. Mathioudakis, J. Yorke, P.R. Williamson, J. Vestbo and J-U. Jensen contributed to study design. A.G. Mathioudakis prepared the initial draft of the manuscript. All authors contributed to critical revision of the paper for intellectual content.

Conflict of interest: A.G. Mathioudakis reports grants from Boehringer Ingelheim outside the submitted work. F. Abroug has nothing to disclose. A. Agusti reports personal fees from AstraZeneca, grants and personal fees from Menarini, personal fees from Chiesi, grants and personal fees from GSK, and personal fees from Nuvaira, outside the submitted work. P. Bakke reports personal fees from GlaxoSmithKline, Chiesi, AstraZeneca and Boehringer Ingelheim outside the submitted work. K. Bartziokas has nothing to disclose. B. Beghe has nothing to disclose. A. Bikov has nothing to disclose. T. Bradbury reports being the recipient of an ongoing 3-year top-up scholarship funded by GlaxoSmithKline. G. Brusselle reports personal fees from AstraZeneca, Boehringer Ingelheim, Chiesi, GlaxoSmithKline, Novartis, Sanofi and Teva, outside the submitted work. C. Cadus reports support from AstraZeneca and Mundipharma, outside the submitted work. C. Coleman is an employee of the European Lung Foundation. M. Contoli reports grants from Chiesi, personal fees from Chiesi, AstraZeneca, Boehringer Ingelheim, ALK-Abello, Novartis and Zambon, and grants from University of Ferrara, outside the submitted work. A. Corlateanu has nothing to disclose. O. Corlateanu has nothing to disclose. G. Criner has nothing to disclose. B. Csoma has nothing to disclose. A. Emelyanov has nothing to disclose. R. Faner reports grants from GSK and Menarini outside the submitted work. G. Fernandez Romero has nothing to disclose. Z. Hammouda has nothing to disclose. P. Horváth has nothing to disclose. A. Huerta Garcia has nothing to disclose. M. Jacobs has nothing to disclose. C. Jenkins has nothing to disclose. G. Joos reports grants and personal fees from AstraZeneca, personal fees from Bayer, grants from Chiesi, personal fees from Eureca vzw, grants and personal fees from GlaxoSmithKline and personal fees from Teva, outside the submitted work; these grants and fees were paid to his institution. O. Kharevich has nothing to disclose. K. Kostikas reports grants, personal fees and nonfinancial support from AstraZeneca, Boehringer Ingelheim, Chiesi, ELPEN, GSK, Menarini and Novartis, grants from NuvoAir, and personal fees from Sanofi, outside the submitted work; and was an employee and shareholder of Novartis Pharma AG until 31 October 2018. E. Lapteva has nothing to disclose. Z. Lazar has nothing to disclose. J.D. Leuppi has nothing to disclose. C. Liddle has nothing to disclose. A. López-Giraldo has nothing to disclose. V.M. McDonald reports grants and personal fees from GSK and AstraZeneca, and personal fees from Menarini, outside the submitted work. R. Nielsen reports grants and personal fees from AstraZeneca and Boehringer Ingelheim, grants from Novartis, and grants and personal fees from GlaxoSmithKline, outside the submitted work. A. Papi reports grants, personal fees, nonfinancial support and other from GlaxoSmithKline; grants, personal fees and nonfinancial support from AstraZeneca; grants, personal fees, nonfinancial support and other from Boehringer Ingelheim; grants, personal fees, nonfinancial support and other from Chiesi Farmaceutici; grants, personal fees, nonfinancial support and other from TEVA; personal fees, non-financial support and other from Mundipharma; personal fees, non-financial support and other from Zambon; personal fees, non-financial support and other from Novartis; grants, personal fees and non-financial support from Menarini; personal fees, non-financial support and other from Sanofi/Regeneron; personal fees from Roche; grants from Fondazione Maugeri; grants from Fondazione Chiesi; and personal fees from Edmondpharma, all outside the submitted work. I. Saraiva has nothing to disclose. G. Sergeeva has nothing to disclose. A. Sioutkou has nothing to disclose. P. Sivapalan reports personal fees from Boehringer Ingelheim outside the submitted work. E. Stovold has nothing to disclose. H. Wang has nothing to disclose. F. Wen has nothing to disclose. J. Yorke has nothing to disclose. P.R. Williamson has nothing to disclose. J. Vestbo reports personal fees from AstraZeneca, grants and personal fees from Boehringer-Ingelheim, personal fees from Chiesi, personal fees from GSK, and personal fees from Novartis, outside the submitted work; and his son is an employee of Chiesi. J-U. Jensen has nothing to disclose.

Support statement: This study is funded by the ERS (ERS TF-2019-12). A.G. Mathioudakis, A. Bikov and J. Vestbo are supported by the NIHR Manchester Biomedical Research Centre. Funding information for this article has been deposited with the Crossref Funder Registry.

\section{References}

1 GBD 2015 Chronic Respiratory Disease Collaborators. Global, regional, and national deaths, prevalence, disability-adjusted life years, and years lived with disability for chronic obstructive pulmonary disease and asthma, 1990-2015: a systematic analysis for the Global Burden of Disease Study 2015. Lancet Respir Med 2017; 5: 691-706.

2 GBD Causes of Death Collaborators. Global, regional, and national age-sex specific mortality for 264 causes of death, 1980-2016: a systematic analysis for the Global Burden of Disease Study 2016. Lancet 2017; 390: $1151-1210$.

3 Vogelmeier CF, Criner GJ, Martinez FJ, et al. Global Strategy for the Diagnosis, Management, and Prevention of Chronic Obstructive Lung Disease 2017 report: GOLD executive summary. Eur Respir J 2017; 49: 1700214.

4 Wedzicha JA, Seemungal TA. COPD exacerbations: defining their cause and prevention. Lancet 2007; 370 786-796.

5 Mathioudakis AG, Janssens W, Sivapalan P, et al. Acute exacerbations of chronic obstructive pulmonary disease: in search of diagnostic biomarkers and treatable traits. Thorax 2020; 75: 520-527.

6 Gayle A, Dickinson S, Morris K, et al. What is the impact of GOLD 2017 recommendations in primary care? - a descriptive study of patient classifications, treatment burden and costs. Int J Chron Obstruct Pulmon Dis 2018; 13: 3485-3492. 
7 Hastie AT, Martinez FJ, Curtis JL, et al. Association of sputum and blood eosinophil concentrations with clinical measures of COPD severity: an analysis of the SPIROMICS cohort. Lancet Respir Med 2017; 5: 956-967.

8 National Institute for Health and Care Excellence. Chronic obstructive pulmonary disease in over 16s: diagnosis and management. NICE guideline (NG115). 2018.

9 Wedzicha JA, Wilkinson T. Impact of chronic obstructive pulmonary disease exacerbations on patients and payers. Proc Am Thorac Soc 2006; 3: 218-221.

10 Wedzicha JAEC-C, Miravitlles M, Hurst JR, et al. Management of COPD exacerbations: a European Respiratory Society/American Thoracic Society guideline. Eur Respir J 2017; 49: 1600791.

11 Bafadhel M, McKenna S, Terry S, et al. Acute exacerbations of chronic obstructive pulmonary disease: identification of biologic clusters and their biomarkers. Am J Respir Crit Care Med 2011; 184: 662-671.

12 Papi A, Bellettato CM, Braccioni F, et al. Infections and airway inflammation in chronic obstructive pulmonary disease severe exacerbations. Am J Respir Crit Care Med 2006; 173: 1114-1121.

13 Collins R, Bowman L, Landray M, et al. The magic of randomization versus the myth of real-world evidence. $N$ Engl J Med 2020; 382: 674-678.

14 Chalmers I, Glasziou P. Avoidable waste in the production and reporting of research evidence. Obstet Gynecol 2009; 114: 1341-1345

15 Rosano GMC. Clinical trial design, endpoints, and regulatory requirements. Handb Exp Pharmacol 2017; 243: 67-78.

16 Mathioudakis AG, Janner J, Moberg M, et al. A systematic evaluation of the diagnostic criteria for COPD and exacerbations used in randomized controlled trials on the management of COPD exacerbations. ERJ Open Res 2019; 5: 00136-2019.

17 Mathioudakis AG, Moberg M, Janner J, et al. Outcomes reported on the management of COPD exacerbations: a systematic survey of randomised controlled trials. ERJ Open Res 2019; 5: 00072-2019.

18 Vollenweider DJ, Frei A, Steurer-Stey CA, et al. Antibiotics for exacerbations of chronic obstructive pulmonary disease. Cochrane Database Syst Rev 2018; 10: CD010257.

19 Walters JA, Tan DJ, White CJ, et al. Systemic corticosteroids for acute exacerbations of chronic obstructive pulmonary disease. Cochrane Database Syst Rev 2014; 9: CD001288.

20 Mathioudakis AG, Chatzimavridou-Grigoriadou V, Corlateanu A, et al. Procalcitonin to guide antibiotic administration in COPD exacerbations: a meta-analysis. Eur Respir Rev 2017; 26: 160073.

21 Kirkham JJ, Clarke M, Williamson PR. A methodological approach for assessing the uptake of core outcome sets using ClinicalTrials.gov: findings from a review of randomised controlled trials of rheumatoid arthritis. BMJ 2017 357: j2262.

22 Kirkham JJ, Gargon E, Clarke M, et al. Can a core outcome set improve the quality of systematic reviews?--a survey of the Co-ordinating Editors of Cochrane Review Groups. Trials 2013; 14: 21.

23 Mathioudakis AG, Sivapalan P, Papi A, et al. The DisEntangling Chronic Obstructive pulmonary Disease Exacerbations clinical trials NETwork: DECODE-NET. Rationale and vision. Eur Respir J 2020; 56: 2000627.

24 Kirkham JJ, Davis K, Altman DG, et al. Core Outcome Set-STAndards for Development: The COS-STAD recommendations. PLoS Med 2017; 14: e1002447.

25 Kirkham JJ, Gorst S, Altman DG, et al. Core Outcome Set-STAndards for Reporting: The COS-STAR Statement. PLoS Med 2016; 13: e1002148.

26 Kirkham JJ, Gorst S, Altman DG, et al. Core Outcome Set-STAndardised Protocol Items: the COS-STAP Statement. Trials 2019; 20: 116.

27 Williamson PR, Altman DG, Bagley H, et al. The COMET Handbook: version 1.0. Trials 2017; 18: 280

28 Klokker L, Tugwell P, Furst DE, et al. Developing an OMERACT Core Outcome Set for Assessing Safety Components in Rheumatology Trials: The OMERACT Safety Working Group. J Rheumatol 2017; 44: 1916-1919.

29 MacLennan S, Williamson PR, Bekema $\mathrm{H}$, et al. A core outcome set for localised prostate cancer effectiveness trials. BJU Int 2017; 120: E64-E79.

30 Fish R, Sanders C, Adams R, et al. A core outcome set for clinical trials of chemoradiotherapy interventions for anal cancer (CORMAC): a patient and health-care professional consensus. Lancet Gastroenterol Hepatol 2018; 3 . 865-873.

31 Hauge CH, Jacobs-Knight J, Jensen JL, et al. Establishing survey validity and reliability for American Indians through 'think aloud' and test-retest methods. Qual Health Res 2015; 25: 820-830.

32 Gale NK, Heath G, Cameron E, et al. Using the framework method for the analysis of qualitative data in multi-disciplinary health research. BMC Med Res Methodol 2013; 13: 117.

33 Diamond IR, Grant RC, Feldman BM, et al. Defining consensus: a systematic review recommends methodologic criteria for reporting of Delphi studies. J Clin Epidemiol 2014; 67: 401-409.

34 Keeley T, Williamson P, Callery P, et al. The use of qualitative methods to inform Delphi surveys in core outcome set development. Trials 2016; 17: 230

35 Guyatt GH, Oxman AD, Vist GE, et al. GRADE: an emerging consensus on rating quality of evidence and strength of recommendations. BMJ 2008; 336: 924-926.

36 Guyatt GH, Oxman AD, Kunz R. GRADE guidelines: 2. Framing the question and deciding on important outcomes. J Clin Epidemiol 2011; 64: 395-400.

37 Mokkink LB, Terwee CB, Patrick DL, et al. The COSMIN checklist for assessing the methodological quality of studies on measurement properties of health status measurement instruments: an international Delphi study. Qual Life Res 2010; 19: 539-549.

38 Lewin S, Glenton C, Munthe-Kaas H, et al. Using qualitative evidence in decision making for health and social interventions: an approach to assess confidence in findings from qualitative evidence syntheses (GRADE-CERQual). PLoS Med 2015; 12: e1001895.

39 Kirwan J, Heiberg T, Hewlett S, et al. Outcomes from the Patient Perspective Workshop at OMERACT 6 J Rheumatol 2003; 30: 868-872.

40 Food and Drug Administration. Public Meeting on CDER Standard Core Sets Clinical Outcome Assessments and Endpoints Grant Program. 2019. https://www.fda.gov/drugs/news-events-human-drugs/public-meetingcder-standardcore-sets-clinical-outcome-assessments-and-endpoints-grant-program-0 Date last accessed: 18 August, 2020.

41 Janssens W, Bafadhel M, Chairs of the CCRC, et al. The CICERO (Collaboration In COPD ExaceRbatiOns) Clinical Research Collaboration. Eur Respir J 2020; 55: 2000079. 